\title{
Reducing mitomycin-C-induced ROS levels in mouse feeder cells improves induced pluripotent stem cell colony growth
}

Fatima M Almenario', Ranelle JL Asi ${ }^{\star, 1,2}$, Sonia D Jacinto \& Ahmad RF Mazahery ${ }^{1,2}$

\section{ABSTRACT}

Chemically defined stem cell culture media are often costly, and the use of mitotically arrested mouse embryonic fibroblasts (MEFs) as feeder cells is a popular and cost-efficient way to maintain induced pluripotent stem cells (iPSCs). However, the commonly used mitotic inhibitor mitomycin-C (MMC) is known to cause cellular metabolic stress. Therefore, our aim was to determine whether such stress in feeder cells indirectly affects iPSC growth during coculture. We report that prolonged exposure to MMC causes metabolic stress in MEFs in the form of oxidative dysregulation. Through optimization of MMC exposure time, we show how to effectively arrest MEFs without inducing oxidative stress, thus promoting significantly better colony growth rates $(p<0.05)$, improved viability and longer periods between passages of iPSCs in coculture.

\section{METHOD SUMMARY}

We tested the effects of $5 \mu \mathrm{g} / \mathrm{ml}$ mitomycin-C treatment at various time exposures, ranging from 20 min to $5 \mathrm{~h}$, on the viability, mitotic activity and reactive oxygen species buildup of the mouse embryonic fibroblast feeder cell line SL10. We selected the optimal exposure time based on effective mitotic inhibition, high viability after 7 days and reduced reactive oxygen species levels. We validated the efficiency of this optimized protocol in maintaining viability and accelerating colony growth of induced pluripotent stem cells.

\section{KEYWORDS}

feeder-dependent culture $\cdot$ induced pluripotent stem cells $\cdot$ mitomycin- $C$ treatment $\cdot$ mouse embryonic fibroblasts $\cdot$ reactive oxygen species

'Institute of Biology, University of the Philippines Diliman Ma. Regidor St., University of the Philippines Diliman, Quezon City, NCR, 1101, Philippines; ${ }^{2}$ Natural Sciences Research Institute, University of the Philippines Diliman - Miranda Hall, P. Velasquez St., University of the Philippines Diliman, Quezon City, NCR, 1101, Philippines; *Author for correspondence: Tel.: +02 9818500 3729; rlasi@up.edu.ph

BioTechniques 68: 270-274 (April 2020) 10.2144/btn-20190118
The need for greater capability in stem cell research remains a challenge in developing countries [1,2]. Although the use of robust biological models enhances such capabilities, local unavailability and the costliness of research products often discourage researchers in budget-tight laboratories. Therefore, the need to establish budget-efficient culture strategies is critical for adapting stem cell-based applications and addresses various emerging scientific problems [2].

The discovery of induced pluripotent stem cells (iPSCs) derived from adult somatic cells is an important breakthrough for stem cell research [3]. Circumventing ethical issues of embryonic stem cell use, iPSCs allowed for generation of patient-specific disease models critical for targeted therapeutics [4]. Cultivation of iPSCs requires a complex pluripotency-maintaining environment. This traditionally involves the use of mouse embryonic fibroblasts (MEFs) as feeder cells $[3,5]$ that provide scaffolding for iPSC attachment and enrich the medium with various growth-enhancing and pluripotency-promoting molecules [5]. Therefore, feeder physiological health is critical for optimal maintenance of iPSCs.

Recent iPSC culture systems involve feeder-free conditions using synthetic adhesion matrices, which minimize exposure of iPSCs to MEF-derived pathogens [6]. However, aside from being costly, most matrices are specifically formulated to work with certain media brands, making experimental optimization difficult. To the contrary, the ease in expanding MEFs provides a near-limitless resource of feeders. Because feeders supply most growth factors needed in iPSC maintenance, they minimize the need for expensive growth factors [7]. One study showed that iPSCs in long-term feederless culture exhibit chromosomal deterioration, whereas the use of the feeder method enhanced chromosomal stability attributing to feeder-promoted Fstl1 signaling [8].

Feeders are mitotically incapacitated using mitomycin-C (MMC) or gamma-irradiation to prevent outgrowing cocultured iPSCs. MMC forms covalent cross-links between DNA strands, whereas irradiation directly causes strand breakage to inhibit DNA replication and arrest the cell cycle [9]. These nonproliferative cells still remain viable and metabolically active for the secretion of important growth factors [5]. Although irradiation can treat larger cell numbers, the equipment and radiation source (Cobalt-60) are costly and impractical to obtain. Although it has been reported that irradiated fibroblasts are more efficient than MMC in maintaining hematopoietic stem cells long term [10], the study employed a relatively high MMC concentration $(50 \mu \mathrm{g} / \mathrm{ml})$. In contrast, another study [9] concluded that after time- and dose-dependent optimization of MMC treatment, feeders are able to maintain hematopoietic stem cells for up to 6 weeks. In parallel to the negative impact of MMC itself on fibroblasts [11,12], reports have also shown that irradiation causes membrane damage and metabolic alterations [13]. Ultimately, the use of MMC-treated MEFs still proves to be a relevant, efficient and economic method for iPSC culture, which could be improved $[8,14,15]$. 


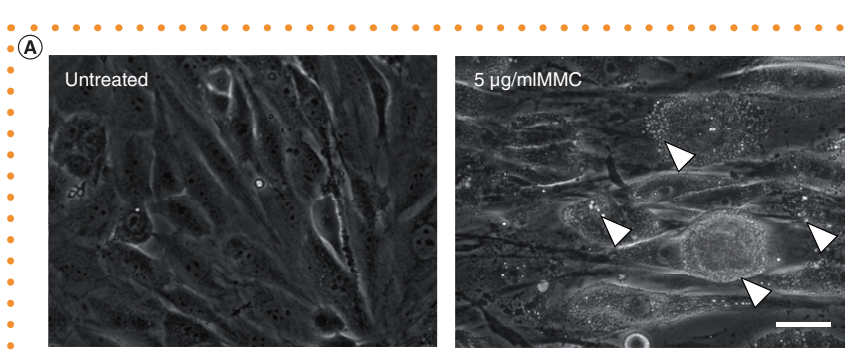

(B)

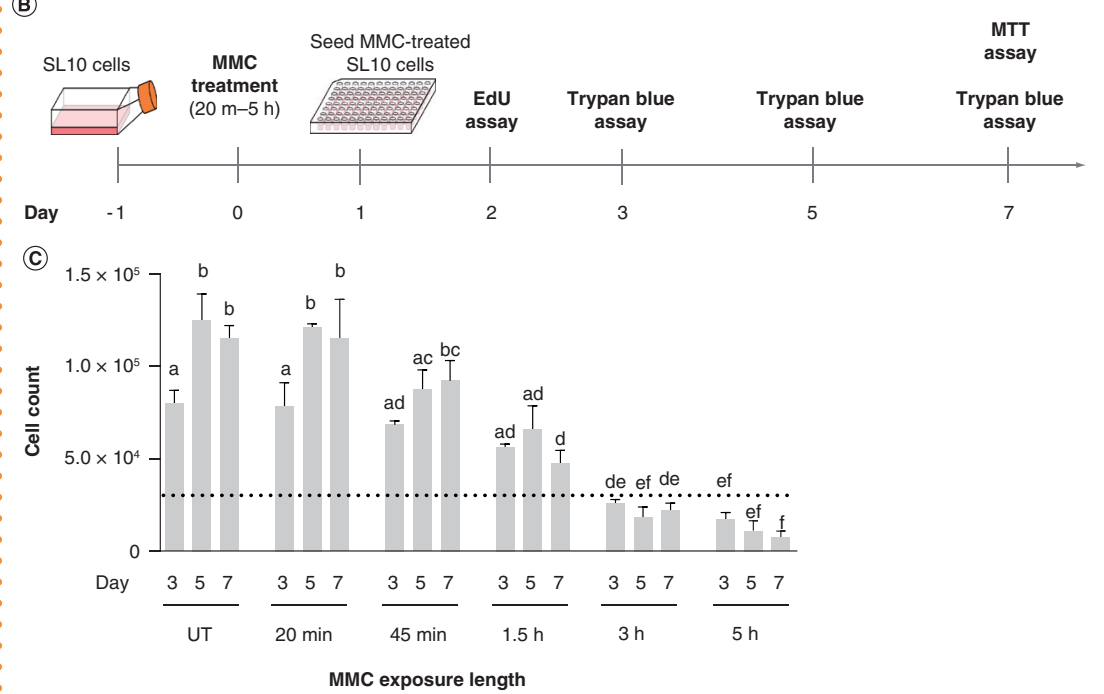

(D)

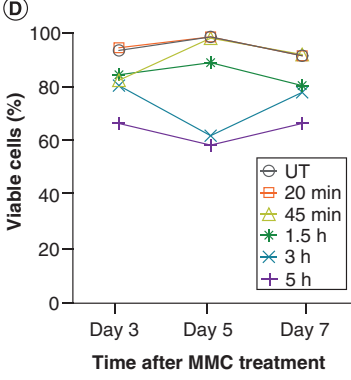

F
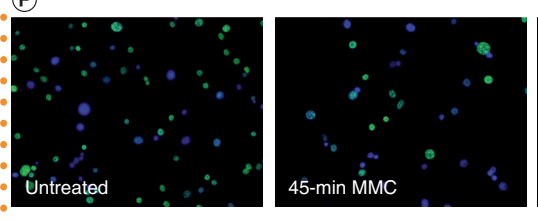

(E)
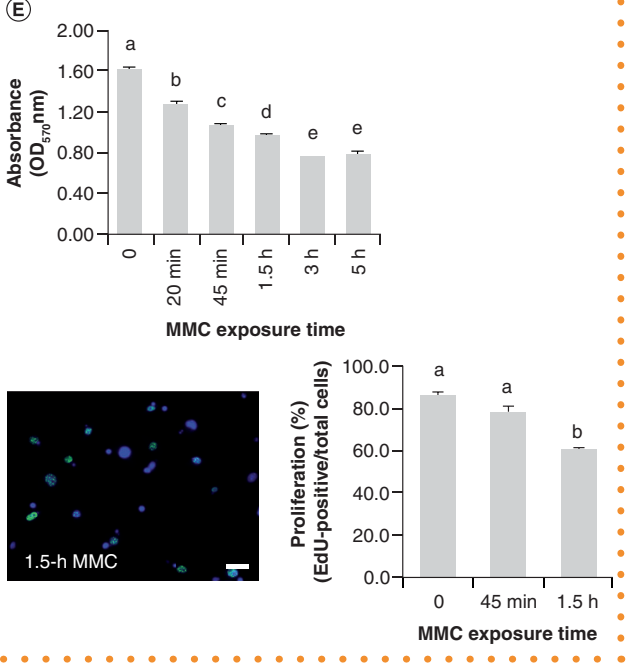

The duration of MMC treatment varies from 2 to $5 \mathrm{~h}[10,11,15]$. Currently, few reports closely examine MMC exposure length as a variable in iPSC maintenance. Therefore, we conducted time-dependent screening of MMC treatment on MEFs to improve their efficiency as iPSC feeders and correlate probable physiological consequences of prolonged MMC exposure to MEF and iPSC growth.

The MEF line SL10 (ReproCell, MD, USA), a derivative of the SIM-derived 6-thioguanine- and ouabain-resistant cells, was used in this study. These were grown in Dulbecco's modified Eagle Medium with 4500 mg/l D-glucose and $2 \mathrm{mM}$ L-glutamine (Thermo Fisher Scientific, OR, USA), supplemented with $10 \%$ fetal bovine serum, $100 \mathrm{U} / \mathrm{ml}$ penicillin, $100 \mu \mathrm{g} / \mathrm{ml}$ streptomycin and $0.25 \mu \mathrm{g} / \mathrm{ml}$ amphotericin B and $0.75 \mathrm{~g} / \mathrm{l}$ sodium bicarbonate (all from Gibco Life Technologies, NY, USA), and maintained at $37^{\circ} \mathrm{C}, 5 \% \mathrm{CO}_{2}$ and $95 \%$ humidity. SL10 experiments without iPSCs were done in either 96 -
Figure 1. Prolonging MMC exposure of SL10 feeders results in significant decrease in cell viability and metabolic activity. (A) MMC-treated cells exhibit atypical morphological changes after 3 days. SL10 feeder cells were treated with $5 \mu \mathrm{g} / \mathrm{ml} \mathrm{MMC}$ for $3 \mathrm{~h}$ and documented for 7 days without media replacement. The left image shows untreated controls exhibiting typical smooth, fibroblast morphology after 7 days. Right image shows MMC-treated SL10 cells showing morphological deformity and heavy cytoplasmic granulation (white arrowheads) after 7 days. Scale bar represents $5 \mu \mathrm{m}$. (B) Timeline of assays performed for time-dependent optimization of MMC treatment of SL10 feeder cells. (C \& D) Cellular viability of SL10 cells decreases as MMC exposure time increases. Cells were treated with $5 \mu \mathrm{g} / \mathrm{ml} \mathrm{MMC}$ at indicated exposure times prior to seeding in a 24-well plate at 30,000 cells per well. Trypan blue assay was performed on the 3rd, 5th and 7th day after MMC treatment. The dotted line indicates the initial density at Day 0 . (E) Metabolic activity decreases as MMC exposure time increases. MTT colorimetric assay was performed on the 7th day after MMC treatment at indicated exposure lengths. (F) MMC exposure for $1.5 \mathrm{~h}$ is enough to significantly lower DNA synthesis in SL10 feeders. Inhibition of DNA synthesis was validated through EdU-Hoechst double staining $24 \mathrm{~h}$ after MMC treatment. Overlaps between blue (Hoechstlabelled nuclei) and green (EdU-labelled newly synthesized DNA strands) were considered positive for DNA synthesis. Scale bar represents $50 \mu \mathrm{m}$. Percent DNA-synthesizing cells was measured through manual count of EdU-positive and total number of cells. For bar graphs - mean values are shown with \pm $\mathrm{SD}$; different letters above the bars indicate statistically significant differences at $p<0.05$ (one-way ANOVA).

ANOVA: Analysis of Variance; EdU-5-Ethynyl2'-deoxyuridine; MMC: Mitomycin-C; MTT: 3-(4,5-dimethylthiazol-2-yl)-2,5-diphenyl tetrazolium bromide; SD: Standard deviation; UT: Untreated.

or 24-well plates at a density of $1.56 \times 10^{4}$ cells $/ \mathrm{cm}^{2}$ (optimized to minimize contact inhibition).

Initially, we observed the effect of MMC exposure on cellular morphology. Changes in SL10 after $5 \mu \mathrm{g} / \mathrm{ml} \mathrm{MMC} \mathrm{(Sigma-Aldrich,}$ MO, USA) treatment for $3 \mathrm{~h}$ were monitored through phase-contrast photomicroscopy over 7 days. Untreated cells retained typical fibroblast morphology after 7 days [16], whereas MMC-treated cells displayed cytoplasmic deformity and heavy granu- 
Figure 2. Prolonged MMC exposure of SL10 feeder cells significantly increases ROS levels and decreases feeder efficiency in maintaining iPSCs. (A) Intracellular ROS levels in SL10 feeder cells significantly increased during prolonged treatment with MMC. ROS levels were measured using DCFDA fluorometric assay. Intensity of fluorescence at $485 / 535 \mathrm{~nm}(\mathrm{Ex} / \mathrm{Em})$ was quantified using Varioskan fluorometer and expressed as fold change relative to untreated control. (B) iPSC colonies cultured with SL10 feeder cells treated with MMC for $3 \mathrm{hrs}$ exhibited slower colony growth compared to those grown in feeders treated for $1.5 \mathrm{hrs}$. Colony sizes were measured at days 2 and 3 through Image $J$ software. (C) iPSC colonies cultured in SL10 feeders treated with MMC for $3 \mathrm{hrs}$ exhibited earlier signs of cell detachment compared to those grown in feeders treated for $1.5 \mathrm{hrs}$. Phasecontrast images were taken using Carl Zeiss AxiovertA.1 inverted microscope. White arrowheads point to colony edges with detaching iPSCs. Scale bar represents $50 \mu \mathrm{m}$. For bar graphs - mean values are shown with $\pm S D$. Different letters above the bars indicate statistically significant difference at $p<0.05$ (one-way ANOVA).

ANOVA: Analysis of Variance; iPSC: Induced pluripotent stem cell; DCFDA: 2',7'-dichlorofluorescin diacetate; MMC: Mitomycin-C; ROS: Reactive oxygen species; SD: standard deviation; TBHP: tert-Butyl hydroperoxide (positive control for ROS). lation by day 3 , which accumulated until day 7 (Figure 1A). Cytoskeletal remodeling is a common stress response in fibroblasts [17], indicating that $3 \mathrm{~h}$ of $\mathrm{MMC}$ exposure caused cellular stress.

To determine whether this cellular stress was attributed to prolonged MMC exposure, we treated SL10 cells with $5 \mu \mathrm{g} / \mathrm{ml} \mathrm{MMC} \mathrm{at}$ various durations (20 $\mathrm{min}, 45 \mathrm{~min}, 1.5 \mathrm{~h}$,
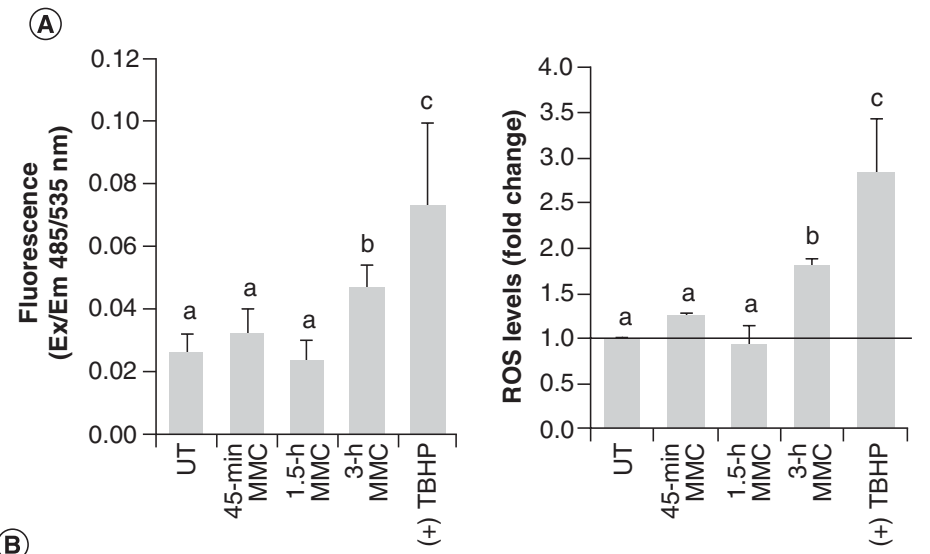

\begin{tabular}{|l|l|r|r|r|}
\hline \multirow{2}{*}{$\begin{array}{c}\text { MMC } \\
\text { exposure } \\
\text { time }\end{array}$} & Trial no. & \multicolumn{1}{|c|}{ Day 2 } & \multicolumn{1}{c|}{ Day 3 } & \multicolumn{1}{c|}{$\begin{array}{c}\text { Colony } \\
\text { growth (\%) }\end{array}$} \\
\cline { 3 - 4 } $\mathbf{1 . 5} \mathbf{~ h}$ & T1 & $50,216.82$ & $123,153.75$ & 245.24 \\
\hline & T2 & $77,477.34$ & $126,871.88$ & 163.75 \\
\hline & T3 & $194,616.00$ & $363,177.00$ & 186.61 \\
\hline & Mean & $\mathbf{1 0 7 , 4 3 6 . 7 2}$ & $\mathbf{2 0 4 , 4 0 0 . 8 7}$ & $\mathbf{1 9 8 . 5 4}$ \\
\hline $\mathbf{3} \mathbf{h}$ & T1 & $54,014.46$ & $80,985.55$ & 149.93 \\
& T2 & $65,119.92$ & $87,904.69$ & 134.99 \\
& T3 & $127,395.00$ & $171,234.00$ & 134.41 \\
\hline & Mean & $\mathbf{8 2 , 1 7 6 . 4 6}$ & $\mathbf{1 1 3 , 3 7 4 . 7 4}$ & $\mathbf{1 3 9 . 7 8}$ \\
\hline
\end{tabular}

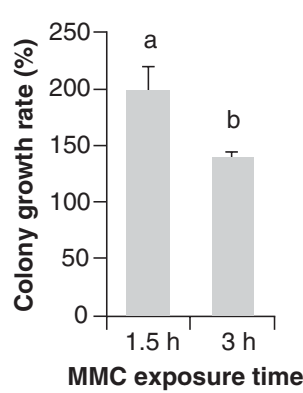

(C)

Day 2

Day 3
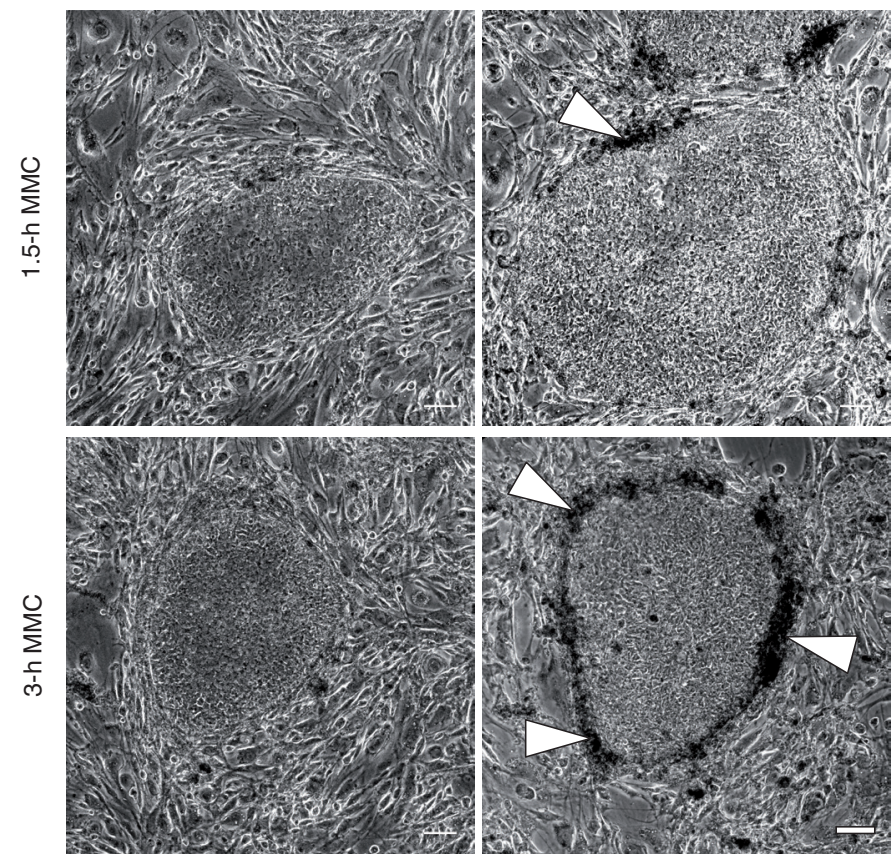

$3 \mathrm{~h}$ and $5 \mathrm{~h}$ ), then measured viability using trypan blue assay on the third, fifth and seventh day post-treatment. Untreated cells, and those treated with MMC for 20 min and MMC for 45 min, remained highly proliferative even after 7 days, suggesting insufficient mitotic incapacitation (Figure 1C). In contrast, despite an initial increase in number, cells treated with $\mathrm{MMC}$ for $1.5 \mathrm{~h}$ had relatively constant counts from day 3 to 7 (Figure 1C) and high viability (Figure 1D), suggesting efficient mitotic inhibition with minimal cytotoxicity. A 3-h MMC treatment was able to efficiently inhibit proliferation based on cell counts (Figure 1C) but resulted in lower viability (Figure 1D). Last, cells treated with MMC for $5 \mathrm{~h}$ exhibited a significant decrease $(p<0.05)$ in total population and viability, 
indicating the cytotoxic effects of prolonged MMC exposure.

Physiological competence of SL10 can be correlated to mitochondrial activity, which was assessed through MTT assay. Compared with the untreated control, less than $50 \%$ metabolic activity was observed after treatment with MMC for $3 \mathrm{~h}$ (Figure 1E), showing the disadvantage of this treatment. In contrast, treatment with MMC for $1.5 \mathrm{~h}$ retained above $50 \%$ metabolic activity after 7 days (Figure 1E). Altogether, the 1.5-h MMC treatment sufficiently kept aconstant feeder population while retaining metabolic competency. To further validate treatment efficiency, we assessed DNA synthesis, an important prerequisite to cell cycle progression, $24 \mathrm{~h}$ post-treatment (Figure 1B). We performed fluorescent labeling using the Click-iT ${ }^{\mathrm{TM}} \mathrm{EdU}$ Alexa Fluor 488 Imaging kit (Invitrogen, CA, USA) according to the manufacturer's protocol and nuclear counter-staining using 1:1000 Hoechst 33342 (Dojindo Molecular Technologies, Kumamoto, Japan). Results show that 1.5-h MMC treatment significantly reduces the proportion of DNA-synthesizing (EdU-positive) cells to $60 \%(p<0.05)$ (Figure 1F), whereas the 45-min MMC treatment showed no statistical difference from untreated control $(p<0.05)$. In agreement with our trypan blue data, 1.5-h MMC treatment incompletely but effectively induces mitotic arrest, which proves ideal in maintaining prolonged viability and metabolic competency of SL10 for 7 days.

Based on morphological symptoms (Figure 1A) and reports of MMC-induced oxidative stress [12,18], we evaluated the involvement of intracellular reactive oxygen species (ROS) buildup in MMC-exposed SL10 cells. We measured ROS using the 2',7'-dichlorofluorescin diacetate assay (Abcam, Cambridge Science Park, UK) following the manufacturer's protocol. ROS levels during the 45-min and 1.5-h MMC treatment did not statistically differ from the untreated group $(p<0.05)$, whereas those in 3-h treatment significantly increased by around 1.6-fold ( $p<0.05$; Figure 2A). This suggests that reduction in metabolic activity in 3-h MMC treatment is not only attributed to mitotic incapacitation, but also to physiological impairment resulting from ROS build up.
Our findings strongly suggest that the differences in viability, metabolic activity and ROS levels between 1.5- and 3-h MMC-treated feeder cells are likely to result in differential ability in iPSC maintenance. Hence, we compared iPSC growth supported by these two feeders. Human iPSC line iPS-TIG114-4f1 (Japanese Collection of Research and Bioresources Cell Bank, National Institute of Biomedical Innovation, Health and Nutrition [NIBIOHN], Osaka, Japan) was seeded on feeders treated with MMC for $1.5 \mathrm{~h}$ or $3 \mathrm{~h}$. SL10 cells were MMC-treated a day prior to seeding in 6-well plates at $3.125 \times 10^{4}$ cells $/ \mathrm{cm}^{2}$ (as recommended by the media manufacturer). The following day, iPSCs were seeded on prepared feeders and allowed to attach for $48 \mathrm{~h}$. Here, we used Primate Embryonic Stem culture medium (ReproCell) containing $5 \mathrm{ng} / \mathrm{ml}$ bFGF (ReproCell), a well-established pluripotency-maintaining agent required in most formulations $[11,15,19]$. This medium was replenished daily. We monitored colony growth and morphology for 5 days. At day 3 , after seeding, we imaged and measured pixel areas of marked iPSC colonies using ImageJ software (Figure 2C). Likewise, we measured the same colonies at day 5 and compared the measurements with day 3 . Results show that iPSC colonies grown in MMC-3 3-h MMC-treated feeders had significantly reduced growth (Figure 2B) and developed signs of detachment around the colony edges (Figure $2 \mathrm{C}$ ). In contrast, those grown in 1.5-h MMC-treated feeders exhibited significantly higher growth $(p>0.05)$ and improved colony morphology. From this, we conclude that prolonged MMC exposure alongside ROS buildup impacts feeder viability and metabolism, which in turn affects iPSC growth. ROS in homeostatic amounts activate pathways associated with regulation of gene expression [20]. However, elevated ROS can induce irreversible damage to biological molecules, leading to cell death or senescence [12]. Here, ROS buildup likely plays a key role in metabolic alterations induced by prolonged MMC exposure in SL10 cells, leading to impairment of their feeder function. In support of this, it has previously been reported that suboptimal feeder conditions indeed lead to apoptosis of pluripotent cells $[11,19]$. Overall, we show that limiting exposure time minimizes
MMC-induced stress on feeder cell function. Therefore, we propose that this simple timedependent optimization, using ROS levels as an indicator, can improve the cost-effectiveness of feeder cells for iPSC maintenance with improved cell yields.

Optimizing time-dependent culture conditions based on parameters such as ROS levels, can improve the yield and timecost expenses of budget-tight laboratories, such as those in developing countries starting their own stem cell facilities.

\section{SUPPLEMENTARY DATA}

To view the supplementary data that accompany this paper please visit the journal website at: www.future-science. com/doi/suppl/10.2144/btn-2019-0118

\section{AUTHOR CONTRIBUTIONS}

FM Almenario and RJL Asi conceptualized and developed the study, collected the data and drafted the manuscript. SD Jacinto provided the facility for laboratory work and reviewed the manuscript. ARF Mazahery oversaw all aspects of the work, including funding allocation, project direction, data and method analysis, and manuscript writing.

\section{ACKNOWLEDGMENTS}

The authors thank the Institute of Resource Development and Analysis, Kumamoto University, for providing the cells used in this study and the Philippine Department of Science and Technology Balik Scientist Program under the office of the Philippine Council for Health Research and Development.

\section{FINANCIAL \& COMPETING INTERESTS DISCLOSURE}

This study was funded by the Natural Sciences Research Institute (grant no. BIO-18-1-05), and the Office of the Vice Chancellor for Research and Development, University of the Philippines Diliman (grant no. 181818PhDIA). The authors have no other relevant affiliations or financial involvement with any organization or entity with a financial interest in or financial conflict with the subject matter or materials discussed in the manuscript apart from those disclosed.

No writing assistance was utilized in the production of this manuscript. 


\section{ETHICAL CONDUCT OF RESEARCH}

All cells used in this study were commercially purchased by IRDA Kumamoto University and therefore required no further clearances to be used for research purposes.

\section{OPEN ACCESS}

This work is licensed under the AttributionNonCommercial-NoDerivatives 4.0 Unported License. To view a copy of this license, visit http://creativecommons.org/licenses/ by-nc-nd/4.0/

\section{REFERENCES}

1. Greenwood HL, Singer PA, Downey GP, Martin DK, Thorsteinsdóttir $\mathrm{H}$, Daar AS. Regenerative medicine and the developing world. PLoS Med. 3(9), e381 (2006).

2. McMahon DS, Thorsteinsdóttir H. Making stem cells count for global health. Regen. Med. 6(Suppl. 6), 163-166 (2011).

3. Takahashi K, Yamanaka S.Induction of pluripotent stem cells from mouse embryonic and adult fibroblast cultures by defined factors. Cell 126(4), 663-676 (2006).

4. Chamberlain S.Disease modelling using human iPSCs. Hum. Mol. Genet. 25, R2R173-R181 (2016).
5. Llames S, García-Pérez E, Meana Á, Larcher F, del Río $M$. Feeder layer cell actions and applications. Tissue Eng. Part B. Rev. 21(4), 345-353 (2015).

6. Fink DW. FDA regulation of stem cell-based products. Science 324, 59351662-1663 (2009).

7. Kueh J, Richards M, Ng S, Chan W, Bongso A. The search for factors in human feeders that support the derivation and propagation of human embryonic stem cells: preliminary studies using transcriptome profiling by serial analysis of gene expression. Fertil. Steril. 85(6), 1843-1846 (2006).

8. Guo R, Ye X, Yang Jet al. Feeders facilitate telomere maintenance and chromosomal stability of embryonic stem cells. Nat. Commun. 9(1), 1-16 (2018).

9. Ponchio L, Duma L, Oliveiro B, Gibelli N, Pedrazzoli , Robustelli della Cuna G. Mitomycin-DNA adducts induce p53-dependent and p53-independent cell death pathways. Cytotherapy 2(4), 281-286 (2000).

10. Roy A, Krzykwa E, Lemieux R, Néron S. Increased efficiency of $\mathrm{Y}$-irradiated versus mitomycin $\mathrm{C}$-treated feeder cells for the expansion of normal human cells in long-term cultures. J. Hematoth. Stem Cell 10(6), 873880 (2001).

11. Nieto A, Cabrera C, Catalina $P$ et al. Effect of mitomycin-C on human foreskin fibroblasts used as feeders in human embryonic stem cells: Immunocytochemistry MIB1 score and DNA ploidy and apoptosis evaluated by flow cytometry. Cell Biol. Int. 31(3), 269-278 (2007).

12. Rjiba-Touati $K$, Ayed-Boussema I, Guedri Y, Achour A,Bacha H,Abid-Essefi S. Effect of recombinant human erythropoietin on mitomycin C-induced oxidative stress and genotoxicity in rat kidney and heart tissues. Hum. Exp. Toxicol. 35(1), 53-62 (2016).

13. Kwon YK, Ha IJ, Bae HW et al. Dose-dependent metabolic alterations in human cells exposed to gamma irradiation. PLOS ONE 9(11), e113573 (2014).

14. Chugh RM, Chaturvedi M, Yerneni LK. An optimization protocol for Swiss 3T3 feeder cell growth-arrest by mitomycin $\mathrm{C}$ dose-to-volume derivation strategy. Cytotechnology 69(2), 391-404 (2017).

15. Li $P$, Wang $S$, Zhan $L$ et al. Efficient feeder cells preparation system for large-scale preparation and application of induced pluripotent stem cells. Sci. Rep. 7(1), 115 (2017).

16. Singhal PK, Sassi S, Lan L et al. Mouse embryonic fibroblasts exhibit extensive developmental and phenotypic diversity. Proc. Natl Acad. Sci. USA 113(1), 122127 (2016).

17. Yamaba H, Haba M, Kunita M,Sakaida T Tanaka H Yashiro Y Nakata S. Morphological change of skin fibroblasts induced by UV Irradiation is involved in photoaging. Exp. Dermatol. 25,45-51 (2016).

18. McKenna E, Traganos F, Zhao H, Darzynkiewicz Z. Persistent DNA damage caused by low levels of mitomycin C induces irreversible cell senescence. Cell Cycle 11, 163132-3140 (2012)

19. Richards $M$, Tan S, Fong $C$ et al. Comparative evaluation of various human feeders for prolonged undifferentiated growth of human embryonic stem cells. Stem Cells 21, 546-556 (2003).

20. Turpaev KT. Reactive oxygen species and regulation of gene expression. Biochemistry (Moscow) 67(3), 281292 (2002). 